\title{
Liquid Lithium Applications for Solving Challenging Fusion Reactor Issues and NSTX-U Contributions
}

\author{
M. Ono ${ }^{1}$, M.A. Jaworski ${ }^{1}$, R. Kaita ${ }^{1}$, Y. Hirooka ${ }^{2}$, T.K. Gray ${ }^{3}$, and the NSTX-U Research Team
}

\begin{abstract}
Steady-state fusion reactor operation presents major divertor technology challenges, including high divertor heat flux both steady-state and transients. In addition, there are unresolved issues of long term dust accumulation and associated tritium inventory and safety concerns [1]. It has been suggested that radiative liquid lithium divertor concepts with a modest lithium-loop could provide a possible solution for these outstanding fusion reactor technology issues, while potentially improving reactor plasma performance $[2,3] . \quad$ The application of lithium (Li) in NSTX resulted in improved H-mode confinement, $\mathrm{H}$-mode power threshold reduction, and reduction in the divertor peak heat flux while maintaining essentially Li-free core plasma operation even during H-modes. These promising results in NSTX and related modeling calculations motivated the radiative liquid lithium (LL) divertor (RLLD) concept [2] and its variant, the active liquid lithium divertor concept (ARLLD) [3], taking advantage of the enhanced non-coronal Li radiation in relatively poorly confined divertor plasmas. It was estimated that only a few moles/sec of lithium injection would be needed to significantly reduce the divertor heat flux in a tokamak fusion power plant. By operating at lower temperatures $\leq 450^{\circ} \mathrm{C}$ than the first wall $\sim 600-700^{\circ} \mathrm{C}$, the LL-covered divertor chamber wall surfaces can serve as an effective particle pump, as impurities generally migrate toward lower temperature LL divertor surfaces. To maintain the LL purity, a closed LL loop system with a modest circulating capacity of $\sim 1$ liter/second $(1 / \mathrm{sec})$ is envisioned to sustain the steady-state operation of a $1 \mathrm{GW}$-electric class fusion power plant. By running the Li loop continuously, it can carry the dust particles and impurities generated in the vacuum vessel to outside where the dust / impurities are removed by relatively simple filter and cold/hot trap systems. Using a cold trap system, it can recover tritium (T) in real time from LL at a rate of $\sim 0.5 \mathrm{~g}$ / sec needed to sustain the fusion reaction while minimizing the $\mathrm{T}$ inventory With an expected $\mathrm{T}$ fraction of $\leq$ $0.7 \%$, an acceptable $\mathrm{T}$ inventory level can be achieved. In NSTX-U [4, 5], preparations are now underway to elucidate the physics of Li plasma interactions with a number of Li application tools and Li radiation spectroscopic instruments. The NSTX-U Li evaporator, which provides $\mathrm{Li}$ coating over the lower divertor plate, can offer important information on the RLLD concept, and the Li granule injector will test some of the key physics issues for the ARLLD concept. A LL loop is also being prepared off-line for prototyping future use on NSTX-U.
\end{abstract}

Keywords: Liquid lithium, tritium recovery, tritium inventory, dust removal, international lithium symposium

\section{Introduction}

Developing a reactor-compatible divertor system is an active area of fusion research for magnetic confinement fusion $[6,7]$. In recent DEMO divertor design studies [8 - 10], the steady-state heat handling capability of a solid-based divertor design is limited to $5-10 \mathrm{MW} / \mathrm{m}^{2}$, which is nearly an order of magnitude lower than the anticipated unmitigated heat flux $\sim 40-60 \mathrm{MW} / \mathrm{m}^{2}$ for the next generation ST-based Fusion Nuclear Science Facility (FNSF) [11], Pilot Plant [12], and a 1 GW-electric-class DEMO/Power Plant with the device size of ITER. In addition, there are serious concerns over potential damage to the PFCs by the very high transient heat fluxes accompanying ELMs and other uncontrolled events [13]. Application of lithium (Li) in the NSTX spherical tokamak [14] resulted in improved $\mathrm{H}$-mode confinement, $\mathrm{H}$-mode power threshold reduction, and ELM mitigation while maintaining essentially Li-free core plasma operation even during H-modes [15-24]. Relatively modest coating of divertor surfaces with lithium (Li) resulted in a factor of two reduction of divertor heat flux in NSTX [25]. A very thin liquid lithium (LL) coating has also protected the surface of the liquid lithium divertor (LLD) substrate [26]. Motivated by this encouraging observation, LL based radiative 
divertor concepts termed the RLLD (radiative liquid lithium divertor) [2] and its variant, the active radiative liquid lithium divertor (ARLLD), have been proposed [3]. In addition to the divertor heat flux issues, there are also important but unresolved fusion reactor technology concerns from long term dust accumulation and associated activation and tritium inventory and safety [1]. A considerable amount of dust or solid particulates is expected to be generated from continuous erosion of the divertor and first wall material due to plasma-material interactions in steady-state fusion power plant operations. If they are allowed to accumulate unchecked, the dust particles (due to the large surface area) could be activated and absorb unacceptable amounts of tritium (T), causing safety and $\mathrm{T}$ inventory issues. With a LL system, it is also necessary to recover $\mathrm{T}$ in real time. In a 1 GW-electric class fusion power plant, about 400 grams of $\mathrm{T}$ is projected to be consumed per day through fusion reactions. The $1 \mathrm{GW}$-electric class fusion power plant challenges are considerably greater than that of ITER due to $4-6$ times fusion power output and high $>70 \%$ operational duty cycle compared to $\leq 5 \%$ for ITER. It is generally estimated that only $\sim 1 \%$ of injected $\mathrm{T}$ is consumed by fusion reactions, the rest being exhausted through the fuel cycle. This means that about $40 \mathrm{~kg}$ of $\mathrm{T}$ must be injected into the fusion power plant per day or about $0.5 \mathrm{~g} / \mathrm{sec}$. Clearly the injected $\mathrm{T}$ must be recovered in real time or the $\mathrm{T}$ site inventory will quickly become unacceptably large. The LL retains $\mathrm{T}$ rather efficiently as LiT (the tritium version of the lithium hydride) and, therefore, the trapped $\mathrm{T}$ in LL must be recovered in real time. In this paper, we explore the possibility of using a LL loop to remove dust and $\mathrm{T}$ from the fusion reactor chamber in real time to solve the dust accumulation and tritium inventory problems. With a modest LL-loop of $1 \mathrm{l} / \mathrm{sec}$ containing only $\sim 0.1 \%$ dust particles by weight, the LL-loop can carry away 16 tons of dust from the reactor chamber annually. With $0.5 \%$ $\mathrm{T}$ by weight, the $1 \mathrm{l} / \mathrm{sec}$ LL-loop can carry away $2.5 \mathrm{~g} / \mathrm{sec}$ $\mathrm{T}$ from the chamber. Since $0.5 \mathrm{~g} / \mathrm{sec}$ of $\mathrm{T}$ is required for the steady-state power plant operation, only about $20 \%$ of $\mathrm{T}$ extraction is therefore required from the LL loop, which makes the $\mathrm{T}$ extraction feasible. In Sec. 2, we give a brief summary of the RLLD and ARLLD concepts. In Sec. 3, the RLLD/ARLLD LL loop system is reviewed. In Sec. 4, a dust removal concept is discussed. In Sec. 5, a cold trap concept for $\mathrm{T}$ extraction is described including a discussion of estimated $\mathrm{T}$ inventory. In Sec. 6, the conclusions and discussions are presented.

\section{Review of Radiative Liquid Lithium Divertor Concepts}

The Radiative Liquid Lithium Divertor / Active Radiative Liquid Lithium Divertor (RLLD/ARLLD) concepts were proposed to reduce the divertor heat flux via radiation of injected lithium in the divertor plasma as shown in Figs 1 and 2. The RLLD/ARLLD are placed at the bottom of the reactor chamber for obvious reasons from the LL handling point of view, and also to capture any impurity particles including dust generated within the reactor chamber [2] as illustrated in Fig. 1. A simplified schematic of the RLLD/ARLLD concept is shown in Fig. 2 , noting that the actual divertor chamber shape should follow the contour of the divertor leg. The LL is introduced at the upper part of the RLLD at multiple toroidal locations, and the LL gradually flows down the RLLD side wall as a thin film via gravity and capillary action. The thin LL film thus formed, spreading over a large surface area, should provide very effective pumping (or entrapment) of the working gases, impurities, and dust generated within the reactor chamber. The RLLD chamber at $\leq 450{ }^{\circ} \mathrm{C}$ being at the lowest temperature in the reactor chamber, together with the usual divertor action, should facilitate the pumping of the entire reactor chamber. It should be noted that since the divertor chamber wall surface area in the fusion power plant is very large i.e., $\geq 100 \mathrm{~m}^{2}$, the LL film may not have to cover the entire surface area of the divertor chamber wall. As shown in Fig. 1, the RLLD chamber wall temperature can be in the $250-450{ }^{\circ} \mathrm{C}$ range, which is significantly lower than that envisioned for the fusion reactor first wall at $\sim 500-700^{\circ} \mathrm{C}$. The hot reactor first wall should be able to keep the wall surfaces clean from tritium and impurities including Li and Li-related compounds [27]. The LL flowing down the divertor side wall accumulates at the bottom of RLLD where the divertor strike point is placed. By placing the LL surface in the path of the divertor strike point, the LL is evaporated from the surface through sputtering, evaporation, and chemical processes [28]. The evaporated Li is quickly ionized by the plasma and the ionized Li ions can radiate strongly in non-coronal fashion in the poorly confined divertor region, reducing the heat flux to the divertor strike point surfaces and protecting the substrate material. The enhanced non-coronal $\mathrm{Li}$ radition has been investigated previously through model calculations $[29,30]$ and also experiments [31-33]. The last line of defense is the LL evaporation from the LLD surface. Through evaporation, Li can carry some heat away from the material surfaces analogous to the way the latent heat of vaporization clamps the temperature rise. The evaporated $\mathrm{Li}$ could also form a $\mathrm{Li}$ vapor cloud in front of the divertor surface and provide some additional protection [34]. The ARLLD concept [3] which is based on active injection of lithium closer to the divertor entrance has the advantage of inducing non-coronal 
radiative loss well away from the divertor plate as shown in Fig 2, thus improving the chance of spreading the heat more evenly throughout the divertor chamber wall. Active Li injection from the divertor side wall also has the advantage of a relatively narrow divertor plasma channel (i.e., short radial travel distance) for Li delivery. The Li therefore can be delivered to the plasma quite rapidly, i.e., $\sim 1 \mathrm{msec}$. Since the particle confinement time of injected $\mathrm{Li}$ in the divertor is estimated to be $\leq 1$ msec even for DEMO parameters, the ARLLD overall response time maybe only $\leq$ a few msec, which should be fast enough to protect the divertor PFCs from transient events.

\section{Liquid Lithium Loop System}

To remove dust / impurities and working gases (i.e., deuterium and tritium), a modest LL circulating loop of $11 / \mathrm{sec}$ was proposed as illustrated in Fig. 3 [2]. This relatively modest level of LL circulation ensures timely removal of generated dust and impurities, including tritium, while keeping the LL purity to be sufficient for smooth LL flow. It should be noted that the LL flow rate of 1 1/sec is much larger (by $10-30$ times) than that required to reduce the heat flux via the RLLD and ARLLD. Therefore most of the circulating LL can be utilized to coat the divertor side wall to provide sufficient pumping for the reactor system. The circulating LL system can also remove dust generated in the reactor chamber which if unchecked can lead to serious activation and $\mathrm{T}$ inventory issues, and related reactor safety concerns. We should note that a similar LL purification loop has been developed for facilities related to the International Fusion Materials Irradiation Facility (IFMIF) [30]. We envision the ARLLD / RLLD LL purification loop for a power plant to have $\mathrm{a} \sim 1000$ liter capacity, which is an order of magnitude smaller than the LL-loop envisioned for IFMIF. The relatively low operating temperature range of the RLLD and its associated LL loop system of $\leq 400{ }^{\circ} \mathrm{C}$ is advantageous from the $\mathrm{Li}$ material corrosion and LL safety point of view. The low operating temperature also makes available broader choices of steel-based alloy materials which might be more practical to employ as a divertor-LL substrate and loop material that is compatible with a reactor environment.

\section{Dust Removal System}

The fusion plasma-wall interactions are known to generate dust or small particles of various sizes [1]. The estimate of dust generation in fusion power plants is not known and its characteristics not yet determined. But what appears to be certain is that such dust will be generated and will likely require its removal. If the dust accumulation is unchecked, it could lead to uncontrolled activation and tritium inventory issues as the total dust surface area could become astronomically large in time. For example, $1 \mathrm{~g}$ of micron-size dust can cover $1 \mathrm{~m}^{2}$ of surface area. The LL loop can provide a means to carry out the dust generated by the plasma. With a $11 / \mathrm{sec}$ LL flow, even a modest $0.1 \%$ dust content by weight means that the LL-loop could still carry nearly 16 tons of dust per year. The LL-loop dust filter concept is shown in Fig. 4. As shown in the figure, the dust filter should be located directly below the divertor as close as practical so that the dust removal can be performed quickly after the LL exits the divertor chamber. With the dust filter located immediately below the divertor chamber, the LL should flow down into the dust filter mostly by gravity, but it may be wise to devise an additional means of moving LL into the dust filter. They can be, for example, a slowly moving screw mechanism facilitating the movement of LL from the divertor exit into the dust filter as illustrated in Fig. 4 and/or a j x B force driven mechanism to move LL within the divertor toward the LL exits [36]. One could remove heavier and larger dust particles by letting the dust to settle at the bottom of the trap, reducing the burden on the dust filter. One would envision several LL loop exits and dust filters distributed toroidally around the torus to insure that at least one of the filters operates at any given time, so that the filled dust filters can be removed without stopping the LL flow. Having a redundancy capability is also wise in case of dust filter failure. The dust filter could also filter any solidified Li compounds formed within the vacuum vessel in addition to the metal-based dust. Since the dust could be trapping tritium, the dust filter should be periodically drained of LL and heated to release any tritium. The released gas can be sent to a conventional tritium separation system to recover tritium for fuel recycling. After the dust filter is filled up, it must be replaced. Since the location of the dust filter is relatively close to the fusion chamber, the dust filter replacement must be done remotely. Once the LL is filtered to be free of dust and other solid materials, it should flow well and only require modest size pipes. This is important to minimize the LL-loop volume as well as the tritium inventory. The filtered LL will be sent to cold traps for tritium removal before returning to the RLLD/ARLD as shown in Figs. 4, 5.

\section{Tritium Removal by Cold Trap}

In a $1-\mathrm{GW}$-electric-class fusion power plant, it is typically estimated that about $0.5 \mathrm{~g}$ of $\mathrm{T}$ is injected into the vacuum vessel per second or about $40 \mathrm{~kg}$ of tritium 
per day. This rate of injection assumes about $1 \%$ fusion burn efficiency, as about $400 \mathrm{~g}$ of T per day or $0.005 \mathrm{~g} / \mathrm{sec}$ of $\mathrm{T}$ is consumed by fusion reactions to generate the required fusion power of $\sim 3 \mathrm{GW}$. Since the amount of injected $\mathrm{T}$ consumed by fusion reactions is very small $(\sim$ $1 \%$ ), it is highly important to recover the remaining $99 \%$ of unused $\mathrm{T}$ in real time to maintain the fuel cycle and keep the plant $\mathrm{T}$ inventory as low as practical. In the IFMIF, the cold traps (CTs) are used mainly to remove oxygen but not tritium since the $\mathrm{T}$ concentration is well below the $\mathrm{T}$ saturation limit of $\sim 0.02 \%$ (by weight) at $200^{\circ} \mathrm{C}$ as noted below. Because of the very low $\mathrm{T}$ concentration, $\mathrm{T}$ was removed using a hot trap [35, 37]. But in the present Li loop system, the $\mathrm{T}$ concentration in the LL is expected to be well above the T saturation limit, so that the recovery of $\mathrm{T}$ with the $\mathrm{CT}$ system is feasible [2]. As for the case of the dust filter system, it is essential to have multiple CT filters for redundancy and regeneration. Once the $\mathrm{CT}$ filter is ready for $\mathrm{T}$ regeneration, the filter is drained of LL. Then the CT is heated up for regeneration and the released $\mathrm{T}$ and other gases are collected and sent to the $\mathrm{T}$ recovery system as in the case of the dust filter. Such a T recovery system has been operational in TFTR and JET, and such a system is needed for any D-T fusion system. Once regenerated, the filter is ready for operation. To minimize the required energy, one can use a heat exchanger as depicted in Fig. 5 to help cool down the LL to the cold trap inlet temperature of $\sim 200^{\circ} \mathrm{C}$ and help reheat the exited $\mathrm{LL}$ to $\sim 300^{\circ} \mathrm{C}$ before returning to the RLLD/ARRD.

The cold trap system for tritium removal - For removing LiT from LL, a cold trap (CT) system appears to be quite energy efficient (particularly with a heat exchanger) since LL is only required to cool down to about $200^{\circ} \mathrm{C}$. The CT uses the property of large changes in the solubility of LiT in LL with temperature as shown in Fig. 6 [33]. At $400{ }^{\circ} \mathrm{C}$, the $\mathrm{T}$ solubility $\mathrm{C}_{\mathrm{CT}}$ is $\sim 0.5 \%$ while the solubility goes down to $\sim 0.02 \%$ at $200{ }^{\circ} \mathrm{C}$. This solubility difference gives the opportunity to extract tritium from LL in the CT. This solubility curve represents the ultimate $\mathrm{CT}$ achievable concentration or $\mathrm{C}_{\mathrm{CT}}$. So, at $200{ }^{\circ} \mathrm{C}, \mathrm{C}_{\mathrm{CT}}$ is $\sim 0.02 \%$ as shown in Fig. 6. It should be noted that in order to recover $0.5 \mathrm{~g} / \mathrm{sec}$ of $\mathrm{T}$, the cold trap has to only reduce the $\mathrm{T}$ concentration in flowing LL of $11 / \mathrm{sec}$ by $0.1 \%$. If the LL flow is doubled to $21 / \mathrm{sec}$, the required $\mathrm{T}$ concentration drop is halved to $0.05 \%$. But higher flow rate means that LL spends much less time in the CT, so the $\mathrm{T}$ recovery efficiency only goes up incrementally with the flow rate as shown below. The $\mathrm{T}$ inventory is lower if the LL LiT concentration is lower but the ability for the CT to remove tritium would improve with higher LiT concentration. So, the actual LiT concentration level maybe determined by the $\mathrm{T}$ inventory and $\mathrm{CT}$ performance considerations. Considering the CT system requirements, the LiT concentration before and after the CT can be expressed in steady-state as

$$
C_{f}=C_{C T}+\left(C_{i}-C_{C T}\right) \exp \left(-\tau_{T r a n} / \tau_{C T}\right)
$$

where $C_{i}$ is the LiT concentration before the $\mathrm{CT}, C_{f}$ is the LiT concentration after the CT, $\tau_{\mathrm{CT}}$ is the cold trap characteristic time constant, and $\tau_{\text {Tran }}$ is the LL transit time in the CT, or $\tau_{\text {Tran }} \sim \mathrm{V}_{\mathrm{CT}} / \mathrm{f}_{\mathrm{LL}}$ where $\mathrm{V}_{\mathrm{CT}}$ is the CT volume and $f_{L L}$ is the LL flow rate in $1 /$ sec. The shorter the $\tau_{\mathrm{CT}}$, the more challenging it is to design the CT system. In order to recover $0.5 \mathrm{~g} / \mathrm{sec}$ of $\mathrm{T}, \mathrm{C}_{\mathrm{i}}-\mathrm{C}_{\mathrm{f}}=$ $0.1 / \mathrm{f}_{\mathrm{LL}}(\%)$. The CT effectiveness tends to go up with $\mathrm{V}_{\mathrm{CT}}$ since $\tau_{\text {Tran }}$ goes up with $\mathrm{V}_{\mathrm{CT}}$, and with larger $\mathrm{V}_{\mathrm{CT}}$, more trapping material can be packed into the CT. The overall CT effectiveness therefore tends to increase rapidly with $\mathrm{V}_{\mathrm{CT}}^{2}$, so $\mathrm{V}_{\mathrm{CT}}$ is a key $\mathrm{CT}$ capability variable. From Eq. 1, we can derive the required $\tau_{\mathrm{CT}}$ as a function of $\mathrm{C}_{\mathrm{i}}$ so that

$$
\tau_{C T}=\tau_{\text {Tran }} / \ln \left[\left(C_{i}-C_{C T}\right) /\left(C_{f}-C_{C T}\right)\right]
$$

As shown in Fig 7 , the $\tau_{\mathrm{CT}}$ increases with increasing $\mathrm{C}_{\mathrm{i}}$, relaxing the $\mathrm{CT}$ requirement. Here, we assumed the $\mathrm{CT}$ volume to be 1001 but it can be changed as required. Also note that for the higher $C_{i}$ range in Fig. 7, the $\tau_{\text {CT }}$ does not vary very much with $f_{L L}$ even though $\tau_{C T}$ is incrementally larger for larger $\mathrm{f}_{\mathrm{LL}}$. For the higher $\mathrm{C}_{\mathrm{i}}$ range, one can reduce Eq. 2 to $\tau_{\mathrm{CT}} \sim 10 \mathrm{~V}_{\mathrm{CT}}\left(\mathrm{C}_{\mathrm{i}}-0.1 /\right.$ $\mathrm{f}_{\mathrm{LL}}-\mathrm{C}_{\mathrm{CT}}$ ), which is relatively insensitive to $\mathrm{f}_{\mathrm{LL}}$, and explains the trend in Fig. 7. As shown in Fig. 7, a 1001 CT can yield $\sim 600 \mathrm{sec}$ or 10 minutes of the characteristic CT time constant, which is within the range of the known CT time constant of this size. If higher CT capability is needed, it can be rapidly increased with the $\mathrm{V}_{\mathrm{CT}}{ }^{2}$ as noted earlier. One of the R\&D issues is the extraction of LiT from the CT. One might want to remove the trapped LiT from CT by increasing the CT temperature above the LiT melting temperature of $\sim 700^{\circ} \mathrm{C}$. Once the LiT is isolated in a special chamber, it may be heated further to its dissociation temperature of $\sim 900^{\circ} \mathrm{C}$. Since the quantity of LiT itself is relatively small, this high temperature treatment might be acceptable. Alternatively, if the LiT can be isolated in a special chamber, one might also consider a chemical means of extracting $\mathrm{T}$ from LiT. For example, $\mathrm{CO}_{2}$ can be introduced to release $\mathrm{T}_{2}$ and form $\mathrm{Li}_{2} \mathrm{CO}_{2}$ (lithium 
carbonate) which is a stable safe compound.

\section{Tritium Inventory of the LL system}

With a LL-loop system, it is important to consider the $\mathrm{T}$ inventory issue for such a system. It is clearly desirable to minimize the $\mathrm{T}$ site inventory. The expected LL volume in a typical LL-loop system in a fusion power plant is depicted schematically in Fig. 8. It is assumed that the LL volume inside the fusion reactor chamber is about 1001 . This is relatively small since the thickness of the LL film for particle pumping purposes can be very thin, i.e., $\sim 0.1 \mathrm{~mm}$ so that the divertor area of $\sim 100 \mathrm{~m}^{2}$ can be covered with only about 101 of LL. For the divertor strike point area for handling heat flux, the LL thickness can be $\sim 1 \mathrm{~mm}$. With the divertor strike point or high heat flux area $\sim 10$ $\mathrm{m}^{2}$, a $1 \mathrm{~mm}$ thick film is only about 101 of LL. For the strike area, it is important to keep the surface covered with LL to protect the solid divertor substrate. The actual equivalent volume of LL within the plasma is negligible for the RLLD and ARLLD. We therefore estimate 1001 to be sufficient for the in-vessel LL volume. We then assume that the LL-loop system up to the entrance of the cold trap volume is 5001 , including the dust filters and connecting pipes. The cold filter and the pipes back to the vacuum vessel, including the circulating pumps and reserve tanks, are assumed to be another 5001 . The total lithium volume is therefore estimated to be 11001 . If the LL volume turns out to be larger or smaller than 11001 , the tritium inventory level will scale accordingly. Assuming a LL flow rate of $11 / \mathrm{sec}$, recoveing $0.5 \mathrm{~g} / \mathrm{sec}$ of tritium in real time for a steady state $1 \mathrm{GW}$-electric fusion power plant operation would then require the $\mathrm{T}$ concentration to be reduced by $0.1 \%$ by the cold trap. In Table 1 , for the $1 \frac{1}{\mathrm{sec}}$ LL-loop system, the $\mathrm{T}$ inventory as a function of the reactor chamber $\mathrm{T}$ concentration in $\%$ is shown to be from $0.3 \%$ to $0.7 \%$. A $\mathrm{T}$ consumption rate of $400 \mathrm{~g}$ per day is assumed. Naturally, the $\mathrm{T}$ inventory increases with the in-chamber $\mathrm{T}$ concentration, but even a $\mathrm{T}$ inventory of 9 days is generally considered to be acceptable. The $\mathrm{T}$ inventory depends only weakly with the LL flow rate. It should be noted that the $\mathrm{T}$ collection is taking place not only in the cold traps but also in the dust filters. As mentioned in Sec. 7, the $\mathrm{T}$ collection should also be taking place in the entire LL-loop system through the double wall configuration. It is important to make sure that $\mathrm{T}$ inventory and transport are well understood and controlled throughout the LL system.

\section{Conclusions and Discussions}

In previous publications, we described the radiative lithium based divertor concepts (RLLD and ARLLD) to solve divertor heat flux issues while improving the plasma performance of fusion reactors. In order to support the RLLD and ARLLD, we proposed a relatively modest LL loop system operating at $\sim 1 \mathrm{l} / \mathrm{sec}$. In this paper, we examined the compatibility of the LL-loop in a fusion power plant including dust, impurity, and $\mathrm{T}$ recovery. The $\mathrm{T}$ recovery from $\mathrm{LL}$ is of particular concern, since timely recovery of $\mathrm{T}$ is crucial to support the $\mathrm{T}$ fuel cycle and maintain the $\mathrm{T}$ inventory at an acceptable level. The previous CT used in the IFMIF related LL-loop facility was mainly used to extract oxygen since the cold trap does not work for very low $\mathrm{T}$ concentration, i.e., $\quad<0.02 \%$ of LL. By operating the T concentration well above the CT limit, the CT can be used to recover LiT for the RLLD/ARLLD LL-loop. For example, with a 1 1/sec LL-loop, the CT only has to reduce the $\mathrm{T}$ concentration by $\sim 0.1 \%$. The $\mathrm{CT}$ efficiency goes up with the LiT concentration level. It might be wise to design the cold trap to operate with lower LiT concentrations, in the $\sim 0.3 \% \mathrm{~T}$ range when it is new. As the cold trap efficiency declines in time with operation, the LiT concentration can be raised accordingly. The CT filter needs to be regenerated to de-trap trapped LiT periodically; therefore the filter material and design must be chosen accordingly to accommodate higher temperature regeneration cycles. We may also note that dust removal using LL could be an important channel for $\mathrm{T}$ recovery as the dust particles could contain significant amounts of $\mathrm{T}$. If the amount of generated dust turns out to be large, the $\mathrm{T}$ released from dust regeneration maybe sufficient to recover most of the $\mathrm{T}$ needed for the $\mathrm{T}$ fuel cycle, reducing the reliance on the cold trap. For this reason, the periodic regeneration of the dust filter for $\mathrm{T}$ release is an essential element of the dust filter design. We also examined the $\mathrm{T}$ inventory issue and concluded that the inventory is likely to be acceptable for a LL-loop system with about a $10001 \mathrm{LL}$ capacity, even with the upper limit of $\sim 0.7 \%$ for $\mathrm{T}$ concentration yielding $3.6 \mathrm{~kg}$ or 9 days of $\mathrm{T}$ inventory. In terms of LL safety, it is important to operate the LL system at or below $\sim 450{ }^{\circ} \mathrm{C}$ to reduce long term corrosion issues, especially since corrosion could accelerate at higher temperatures. Another important consideration is a double walled configuration with an evacuated outer layer as shown in Fig. 8. The vacuum layer provides good thermal insulation to keep the LL hot. It also helps detect LL leaks relatively quickly and provides a LL safety barrier. Quite importantly, the vacuum layer could also provide a means of recovering $\mathrm{T}$ which could be diffused through the hot LL pipe wall [39], contributing to T inventory control.

As the NSTX-U device is starting its operation with 
various lithium tools and related diagnostic systems, it is now possible to investigate pertinent physics issues related to radiative liquid lithium divertor concepts. The NSTX-U Li evaporator system, which provides Li coatings over the lower divertor plate, can offer important information on the RLLD concept, and the Li granule injector [40] will test some of the key physics issue for the ARLLD concept. In particular, the actual lithium radiation level achievable in edge and divertor plasmas per injected lithium particles $[41,42]$ is critical to better quantify the amount of lithium needed to reduce the divertor heat flux to an acceptable level. A LL-loop is also being prepared off line for prototyping future use on NSTX-U. A manageable aspect of the LL-loop development is that the required $R \& D$ s can be performed with a relatively modest laboratory setting where various aspects of the LL-loop such as the dust filter, cold trap, and tritium recovery (using hydrogen or deuterium), etc., can be performed separately.

\section{Acknowledgements:}

One of the authors (MO) appreciates the discussions and suggestions by Drs. H. Kondo, R. Maingi, R. Goldston, C.H. Skinner, and R. Majeski. This work was supported by DoE Contract No. DE-AC02-09CH11466.

[1] Federici G., Skinner C.H. et al., 2001 Nucl. Fusion 411967

[2] Ono M. et al., 2013 Nucl. Fusion 53113030

[3] Ono M. et al., 2014 Fusion Engineering and Design 892838

[4] Menard J.E. et al 2012 Nucl. Fusion 52083015

[5] Ono M. et al., 2015 Nucl. Fusion 55073007

[6] Magnetic Fusion Energy Science Research Needs Workshop report, June 2009, http://burningplasma.org/web/ReNeW/ReNeW.rep ort.web2.pdf

[7] ITER Physics Basis Expert Groups on Confinement and Transport and Confinement Modelling and Database, ITER Physics Basis Editors 1999 Nucl. Fusion 392175

[8] Tobita K. et al 2009 Nucl. Fusion 49075029

[9] Visca E. et al 2012 Fusion Eng. Des. 87941

[10] Wang X.R. et al., 2012 Fusion Eng. Des. 87732

[11] Peng Y-K. M. et al 2009 Fusion Sci. Technol. 56 957

[12] Menard J. E. et al 2012 Nucl. Fusion 52083015

[13] Nygren R.E. et al 2011, J. Nucl. Materials 417451
[14] Ono M. et al 2000 Nucl. Fusion 40557

[15] Kugel H.W. et al 2008 Physics of Plasmas 15 056118

[16] Bell M.G., et al 2009 Plasma Phys. Control. Fus. 51124054

[17] Maingi R. et al 2009 Phys. Rev. Lett. 103075001

[18] Maingi, R. et al 2011 Nucl. Fusion 521823

[19] Kaye S.M. et al 2013 Nucl. Fusion 53063005

[20] Kaye S.M. et al 2012 Nucl. Fusion 51083001

[21] Maingi R. et al 2010 Phys. Rev. Lett. 105135004

[22] Podesta M. et al 2012 Nuclear Fusion 52033008

[23] Scotti F. et al 2013 Nucl. Fusion 53083001

[24] Ono M. et al 2010 Fusion Eng. Des. 85882

[25] Gray T.K. et al 2014 Nucl. Fusion 54023001

[26] Kugel H.W. et al 2012 Fusion Eng. Des. 871724

[27] Capece A. et al., 2015 J. Nucl. Materials 463, 1177

[28] Doerner R.P. 2001 J. Nucl. Matter 290-293 166

[29] V.A.Etikhin et al. Plasma Phys. Contr. Fus. 44 (2002) 955

[30] Rognlien T.D. and Rensink, M.E. 2002 Physics of Plasmas 92120

[31] S.V.Mirnov J.Nucl.Mater. 390-391 (2009) 876

[32] M.L.Apicella et al. Plasma Phys. Contr. Fus. 54 (2012) 035001

[33] L Mirnov S.V., Belov A.M., Djigailo N.T., Kostina A.N., Lazarev V.B., Lyublinski I.E. et al 2013 J. Nucl. Matter S224-S228 438

[34] Jaworski M. et al., 2013 Plasma Phys. Control. Fusion 55124040

[35] Kondo H. et al 2011 Fusion Eng. Des. 862437

[36] Shimada M. and Hirooka Y. 2014 Nucl. Fusion 54 122002

[37] Yagi J. et al 2011 Fusion Eng. Des. 862678

[38] Natesan K. 1983 Journal of Nuclear Materials 115251

[39] Hirooka Y. et al., 2012 Fusion Science and Technology 64345

[40] Mansfield D. et al., 2013 Nucl. Fusion 53113023

[41] Rognlien T.D. and Rensink, M.E. 2002 Physics of Plasmas 92120

[42] Mirnov S.V. et al 2006 Plasma Phys. Control. Fusion 48821 


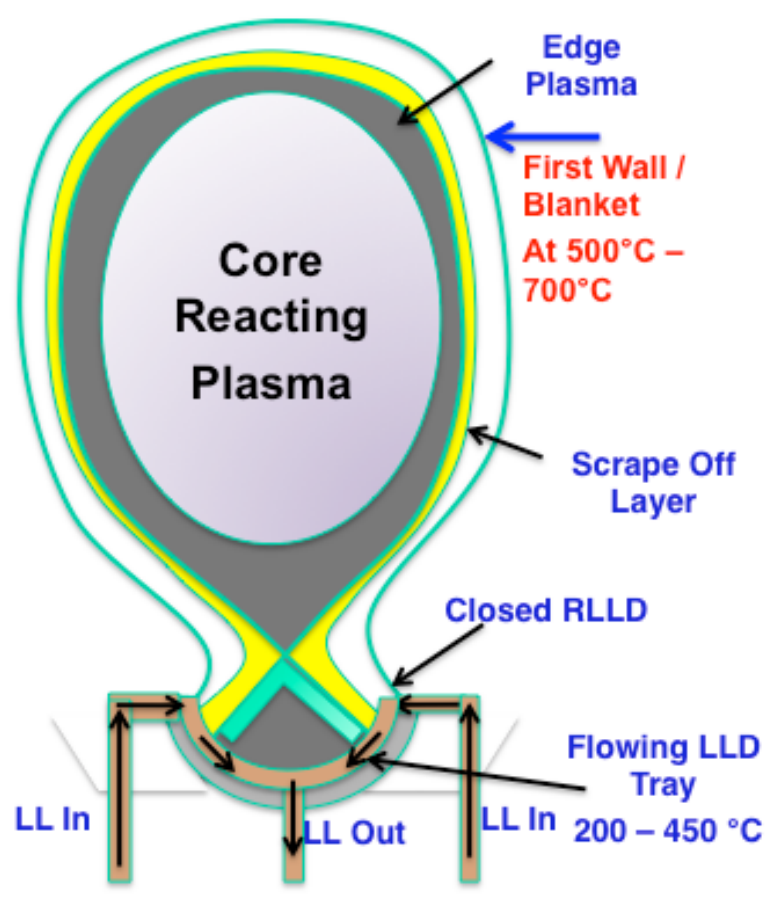

Fig. 1. A possible RLLD configuration in a fusion power plant. The RLLD is envisioned to be placed at the bottom of the reactor chamber to capture LL, dust, and other solid impurities.

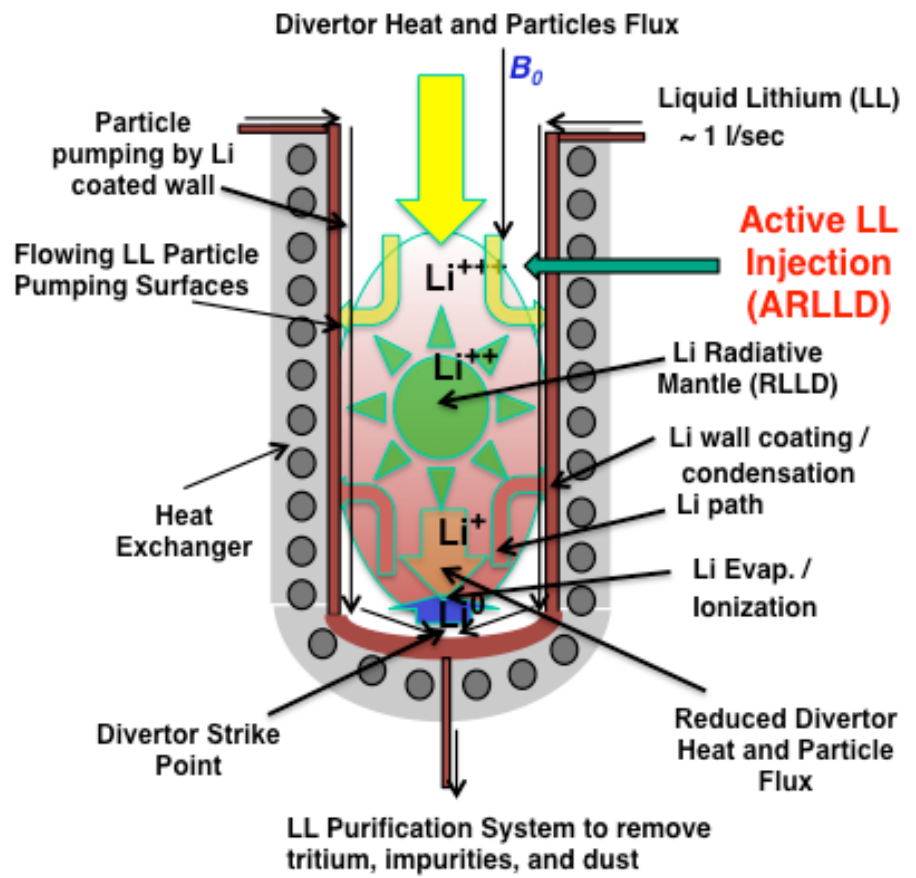

Fig. 2. A simplified schematic of RLLD chamber. The LL flows down along the side wall to provide pumping and the thicker LL layer at the bottom provide radiative Li source for heat flux reduction and divertor substrate protection. The active LL injection from the side wall is also shown. 


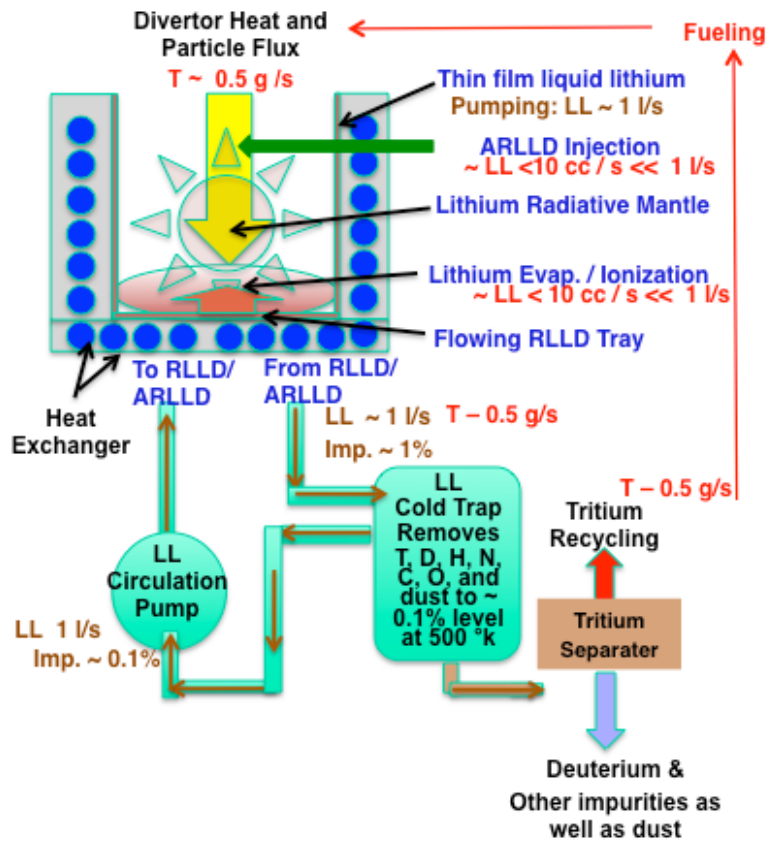

Fig. 3. A simplified schematic for the LL purification loop for RLLD in a fusion power plant.

\section{Divertor Heat and Particle Flux}

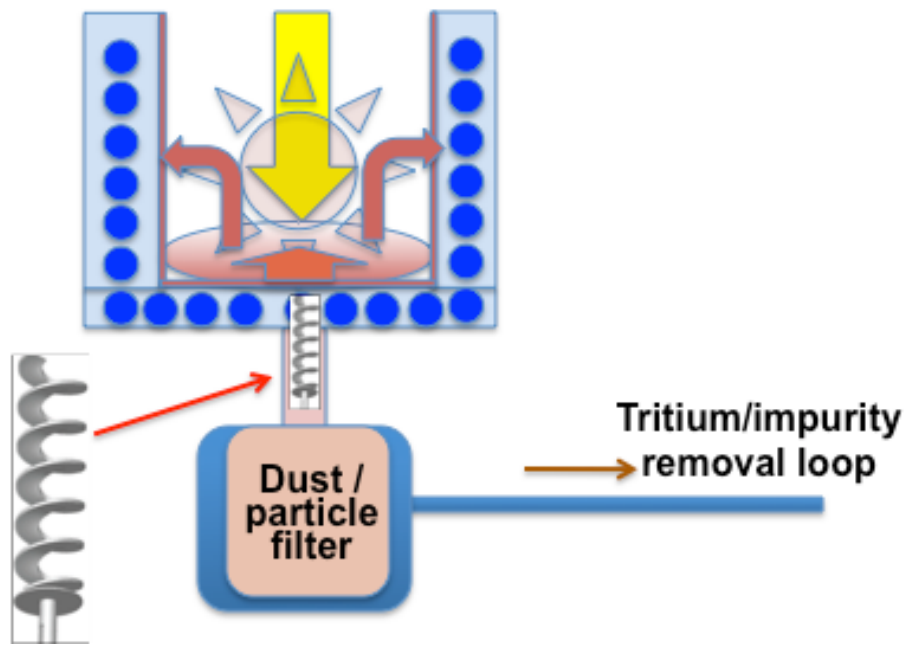

Fig. 4. A schematic for the dust filter placed directly below the RLLD/ARLLD divertor chamber. An illustration of a screw mechanical device is shown to help facilitate the LL movement from divertor chamber to the dust filter. 


\section{From dust/particle filter}

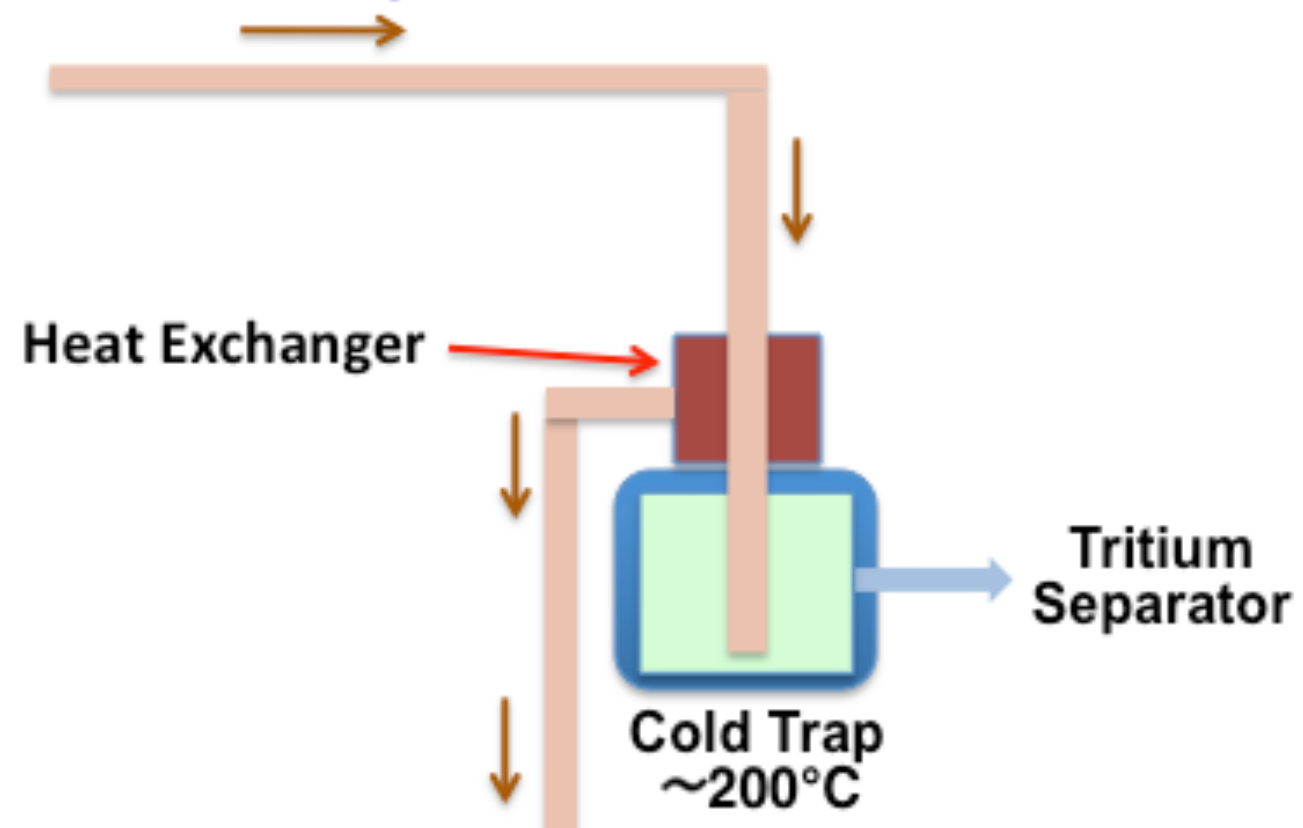

To RLLD/ARLLD

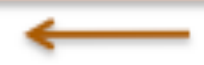

Fig. 5. A simplified schematic of the cold trap within the LL loop system. A possible heat exchanger is shown to minimize the energy requirement to cool the LL to the cold trap temperature.

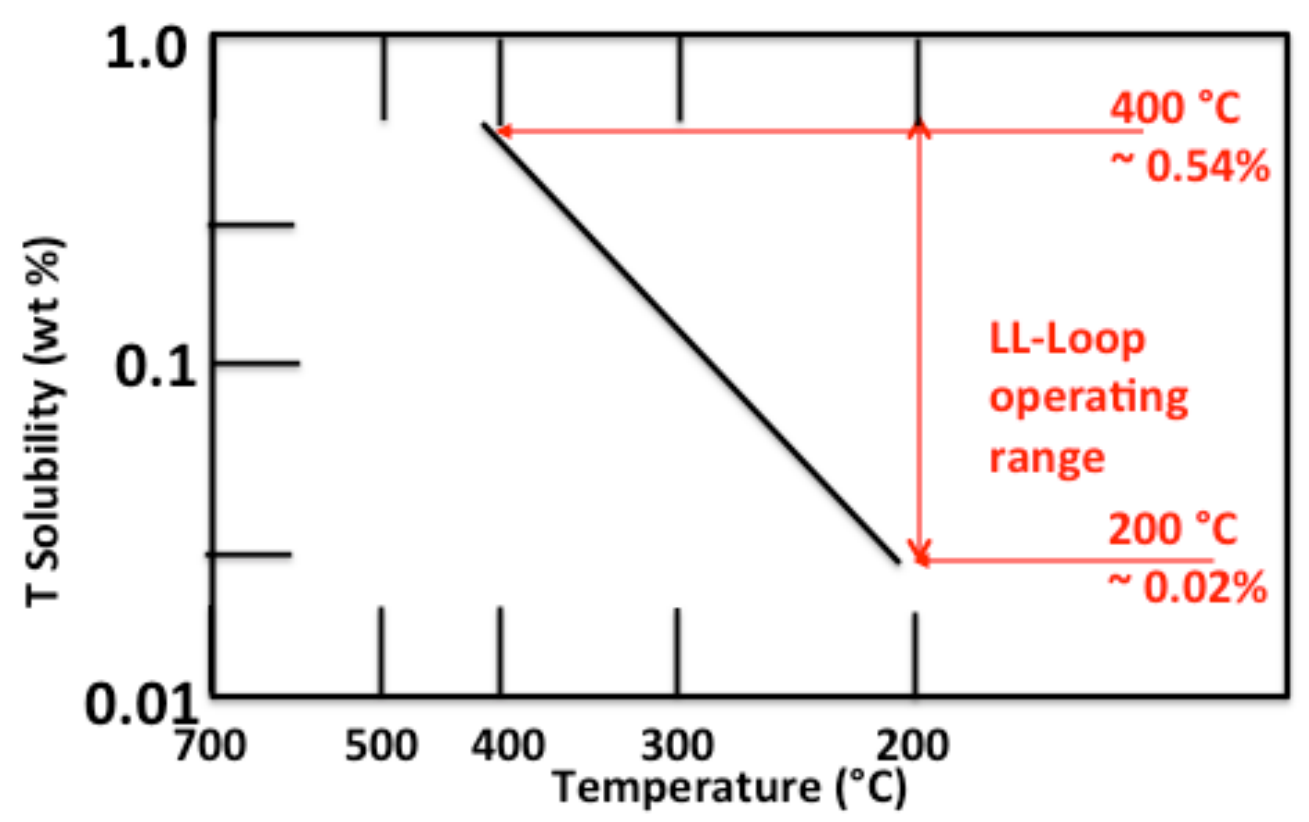

Fig. 6. Tritium solubility vs. liquid lithium temperature. 


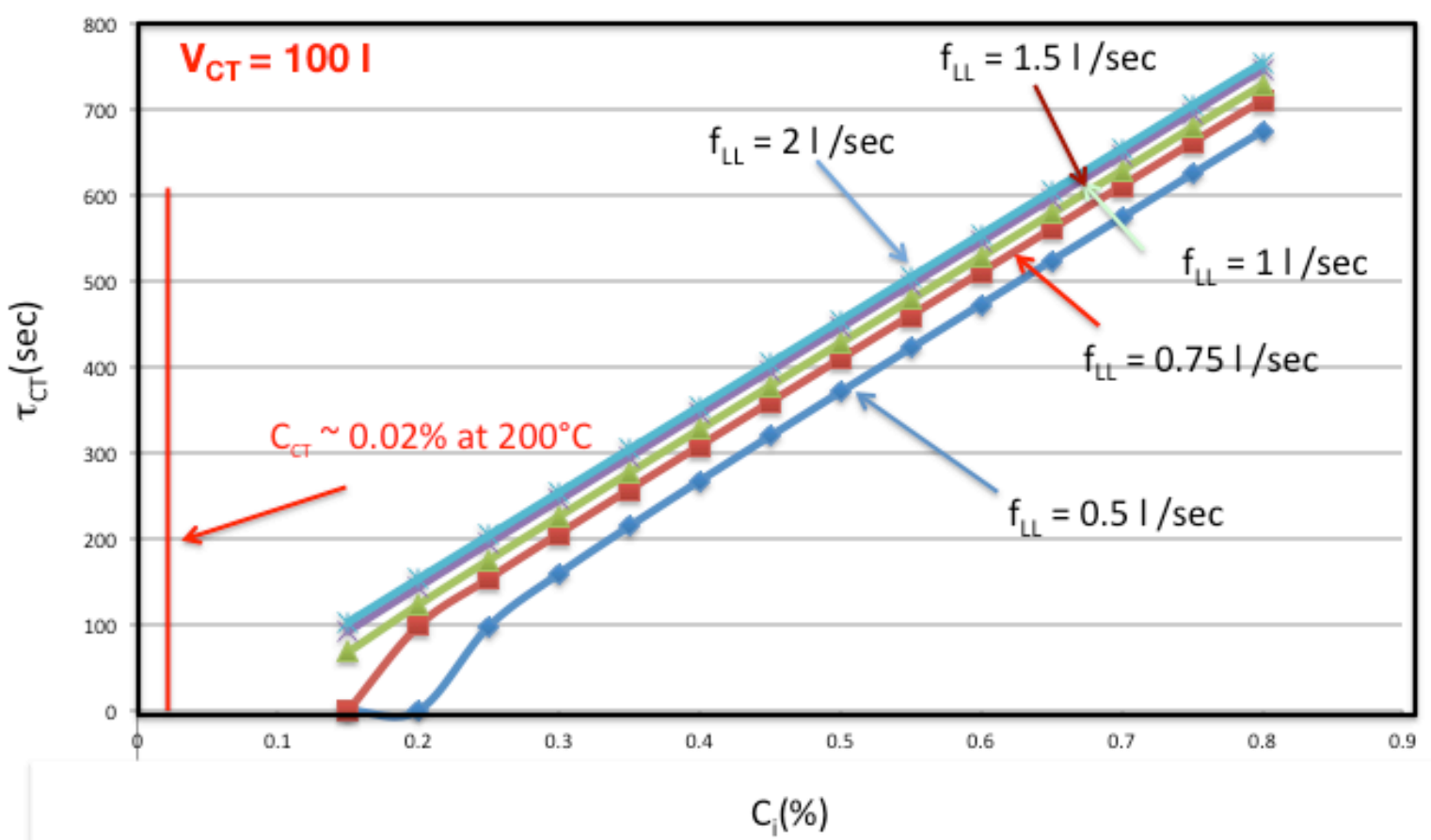

Fig. 7. Characteristic cold trap time vs. tritium concentration in liquid lithium for various liquid lithium flow speed. The assumed cold trap volume is 1001 operated at $200{ }^{\circ} \mathrm{C}$.

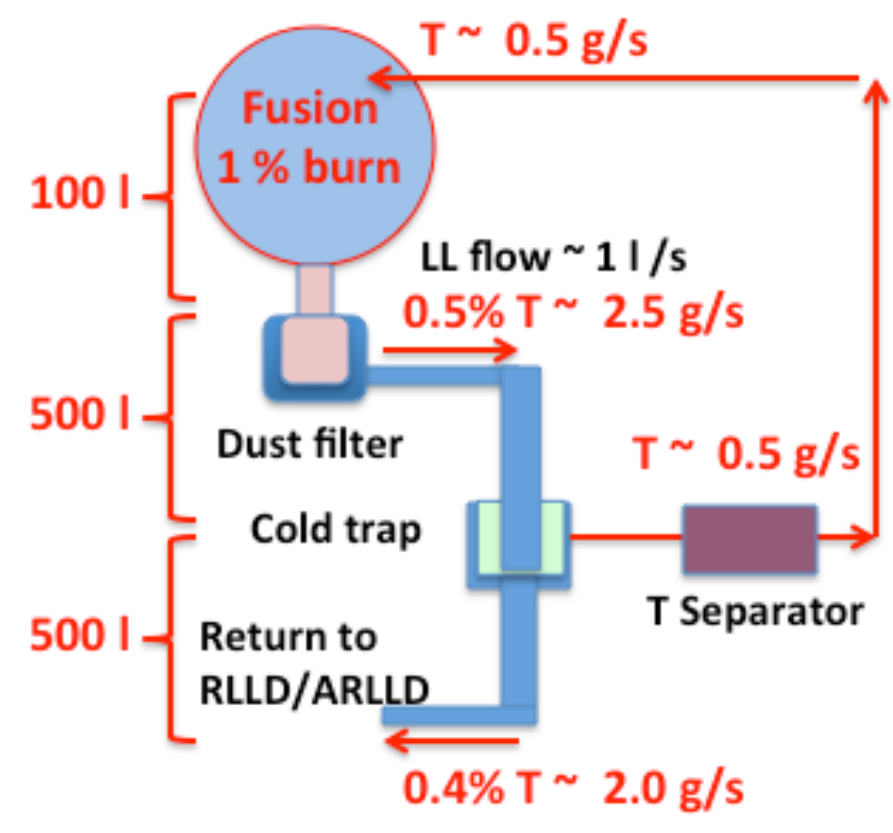

Fig. 8. A schematic of tritium inventory of the LL-loop system. 


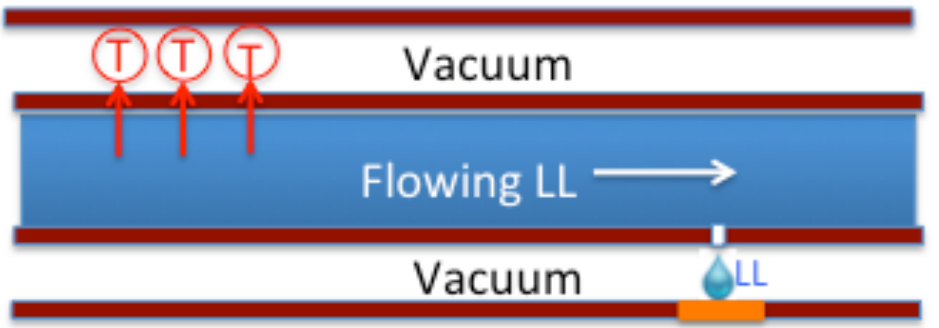

Fig. 9. A double walled LL-loop system to provide added barrier for LL safety, thermal insulation, and tritium recovery.

\begin{tabular}{|c|c|c|}
\hline $\mathrm{T} \%$ & $\mathrm{~T}(\mathrm{~kg})$ & Days \\
\hline 0.7 & 3.6 & 9 \\
\hline 0.6 & 3.1 & 7.6 \\
\hline 0.5 & 2.5 & 6.3 \\
\hline 0.4 & 2.0 & 4.9 \\
\hline 0.3 & 1.4 & 3.5 \\
\hline
\end{tabular}

Table 1. Tritium concentration and tritium inventory.

The Days are assuming 400g tritium consumption per day. 